\title{
Der antimuslimische Rassismus und sein Sicherheitsdiskurs
}

\section{Traditionen des Sicherheitsdiskurses}

Es greift zu kurz, 9/11 als Kristallisationspunkt des antimuslimischen Rassismus in den USA, Europa, Deutschland und anderen Teilen der Welt zu setzen, wie dies häufig geschieht. Anders als es das mediale und politische Sprechen über Islam, Migration und Integration oder auch einzelne Studien aus der Vorurteilsforschung zu Islamophobie suggerieren, hat die Problematisierung der Minderheit und ihre Diskursivierung als bedrohlich und fremd eine lange Tradition.

Gegenwärtige Maßnahmen zur inneren und äußeren Sicherheit, die sich in spezifischer Weise gegen und an (als) Muslim:innen (Markierte) richten, reichen bis ins späte 15. bzw. frühe 16. Jahrhundert in Südeuropa zurück. Auf der Iberischen Halbinsel etwa müssen die über Jahrhunderte ansässigen Bevölkerungsgruppen muslimischen Glaubens und arabischer oder amazighischer Sprachen nach der Machtübernahme durch katholische, spanische und portugiesische Herrscher:innen zunächst verschiedene restriktive Maßnahmen über sich ergehen lassen, die sie in der Ausübung ihrer Religion und an der Entfaltung ihrer Kulturen hindern: Sie werden zur Konversion gezwungen oder vertrieben und schließlich nach Nordafrika deportiert. ${ }^{1}$ Die Idee der neuen, christlichen Herrscher:innen, die Einheit der Nation durch religiöse Reinheit zu erreichen, koppelt die Loyalität 
gegenüber der Nation an die Religionszugehörigkeit, die wiederum genealogisch hergeleitet wird. Der eigenen Logik und Politik zufolge kann die muslimisch-arabisch-amazighische Bevölkerung, >Mauren` oder >Moriscos ～genannt, nicht loyal sein und wird deswegen als gefährlich behandelt, beobachtet, reglementiert, sanktioniert und schließlich vertrieben und deportiert. Die über mehrere Jahrhunderte andauernde Koexistenz der multilingualen und multireligiösen Gesellschaft, die in gemeinsamen Arbeiten philosophische und naturwissenschaftliche Höhenflüge erreicht, nimmt ein gewaltsames Ende. Der vormoderne Rassismus erhält seine Konturen. ${ }^{2}$

Im Zuge des europäischen Kolonialismus verändert sich die Wahrnehmung und Darstellung der >Mohammedaner:innen $<$ im >Orient $<$ und in >Afrika<, die Deutung ihrer Gefährlichkeit erfährt eine neue Variante und geht abermals mit ihrer Rassifizierung einher. Um die koloniale Herrschaft, Gewalt, Ausbeutung, Unterdrückung und Herabwürdigung $\mathrm{zu}$ rechtfertigen, werden die kolonialen >Untertanen und unzivilisiert diskursiviert, der Widerstand dagegen als Aufstand und Gewalt diffamiert. ${ }^{3}$ Die Ver-anderung und die Gewalt sind geblieben, ihre Begründung und ihre Formen verändern sich, wie Fanon argumentiert:

»Der vulgäre, primitive, gewöhnliche Rassismus gab vor, in der Biologie die materielle Basis der Doktrine zu finden, als sich die Bibel als unzureichend erwiesen hatte. [...] Nichtsdestoweniger feiern die alten Äußerungen hier und dort Wiederauferstehung. [...] Der Rassismus, der sich rational, individuell, genotypisch und phänotypisch determiniert gibt, verwandelt sich in einen kulturellen Rassismus. Das Objekt des Rassismus ist nicht länger der einzelne Mensch, sondern eine bestimmte Existenz-Form. [...] Die >westlichen Werte treffen sich seltsamerweise wieder mit dem so berühmten Aufruf zum Kampf des >Kreuzes gegen den Halbmond . $^{4}$

\footnotetext{
$2 \quad$ Hering Torres 2006.

3 Fanon 1966, 1969; Said 1978.

4 Fanon 1969: 39f.
} 
Fanons und Saids Studien zur kolonialen Gewalt und zum Orientalismus nehmen französische und englische Diskurse und Politiken in den Blick. Auch im deutschen Kolonialismus bis hin zum Nationalsozialismus spielt neben der biologischen und kulturellen Orientalisierung der Muslim:innen ihre politische Beherrschung und Instrumentalisierung eine Rolle. ${ }^{5}$ Die Frage danach, welche Gefahr von ihnen ausgeht, bezieht hier, ähnlich wie in anderen europäischen Kontexten, sowohl vormoderne als auch moderne Argumentationslinien ein ${ }^{6}$, die in aktuelle Diskurse hineinragen. Sie bringen ein spezifisches >Wissen Muslim:innen hervor ${ }^{7}$ und verdichten sich in einem neuen antimuslimischen Rassismus. ${ }^{8}$ Darin kommen dem Bedrohungsszenario und dem Sicherheitsdiskurs wieder prominente Bedeutungen $\mathrm{zu}->$ Muslim:innen werden als innere und als äußere Gefahr diskursiviert. ${ }^{9}$

Der aktuelle antimuslimische Rassismus nimmt sowohl auf den vormodernen als auch auf den modernen Rassismus Bezug, sodass einige ältere Topoi erstaunlich aktuell erscheinen - oder auch gegenwärtige, als menschenrechtliche >Islamkritik^präsentierte Äußerungen auffallend mittelalterlich. Das Wechselspiel von Religion, Kultur, Geschlecht und Sexualität etwa blickt auf eine lange Geschichte zurück ${ }^{10}$, aktuelle Analysen $\mathrm{zu}$ Homonationalismus und Femonationalismus ${ }^{11}$ können auf Vorarbeiten zum »kolonialen Feminismus « ${ }^{12}$ zurückgreifen.

Die Vorstellung einer Gefahr, die von Muslim:innen - und auch Juden:Jüdinnen - als >fünfter Kolonne Nation einer äußeren Macht zuarbeitet, finden wir ausgeprägt im 16., im ausgehenden 20. sowie im beginnenden 21. Jahrhundert wieder,

Attia 2007; Berman 1996; Fuhrmann 2006; Keskinkılıç 2019a; Motadel 2017; Wagenhofer 2010.

6 Bellmann 2011; Höfert 2003; Katzer 2008.

7 Amir-Moazami 2018; für Europa: Johansen/Spielhaus 2012.

8 Attia 2009; für Europa und USA: Attia/Keskinkılıç 2016.

9 Benz 2012.

10 Kabbani 1993; Pinn/Wehner 1995; Shooman 2014; Yeğenoğlu 1998.

11 Zum Homonationalismus Puar 2007; zum Femonationalismus Farris 2011.

12 Ahmed 1992: 151. 
während die angebliche Gefahr, die von der vermeintlichen Rückständigkeit und dem Widerstand der Muslim:innen (und der anderen ausgebeuteten Völker) gegen Kolonialismus und die >Neue Weltordnung ausgeht, das 18. und 19. mit dem 21. Jahrhundert verbindet. Die Transformation spiegelt die Entwicklung des Rassismus: Während der vormoderne Rassismus $>$ Rassen im Plural als getrennte Entitäten konzipiert, führt der moderne Rassismus die vormals getrennten $>$ Rassen zusammen - die Differenz wird nun linear in der Entwicklung der Menschheitsrasse, >Rasse $<$ im Singular also, angeordnet. Insofern verwundert es nicht, wenn im vormodernen Rassismus die Gefahr auch dann als äußere wahrgenommen wird, wenn diejenigen, von denen die Gefahr ausgehen soll, im Inneren leben (Illoyalität), während die Gefahr im modernen Rassismus als universalistische, weil den Fortschritt der Menschheit bzw. die >Rassenhygiene zipiert wird (Minderwertigkeit). In jeweils angepasster Sprache und Bezügen finden wir beide Diskursstränge in aktuellen Rassismen, auch im antimuslimischen, wieder. >Muslim:innen sind demzufolge sowohl gefährlich, »weil sie wirklich im Weltmaßstab operieren " $^{13}$ und »an der Übernahme der Macht « ${ }^{14}$ arbeiten, als auch, weil sie aufgrund ihrer »enorme[n] Fruchtbarkeit [...] auf lange Sicht eine Bedrohung für das kulturelle und zivilisatorische Gleichgewicht [und einen] Rückschritt « ${ }^{15}$ darstellen. Insofern verbinden sich im antimuslimischen Rassismus Argumentationsmuster des Antisemitismus mit jenen des kolonialen Rassismus, weisen aber auch Unterschiede zu beiden auf und stellen daher eine spezifische Form von Rassismus dar. ${ }^{16}$

Der kurze Abriss zeigt, dass der Sicherheitsdiskurs nicht neu ist und die Vermutung, dass er auf die Gewalt reagiert, die von (als) Muslim:innen (Markierten) ausgeht, zu kurz greift. ${ }^{17}$

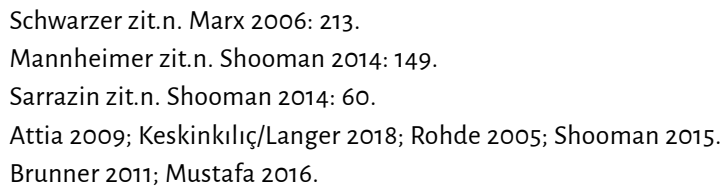




\section{Antimuslimischer Rassismus als Gesellschafts- und Erfahrungshintergrund}

Die Stereotypisierung, Diskriminierung und Verfolgung von (als) Muslim:innen (Markierten) damit zu entschuldigen, dass es nicht die Falschen trifft, angesichts von Terrorismus und Radikalisierung, Illoyalität und Fremdheit, Rückständigkeit und Traditionalismus, verkennt die historischen und gesellschaftlichen Zusammenhänge und bedient sich genau jener Argumentationen, die sie zu kritisieren vorgibt. AlAzmeh vergleicht Die Islamisierung des Islam, so der Titel seines Buches, in europäischen Diskursen mit der "Islamisierung des Islam ${ }^{18}$ durch islamistische Ideologien. Demnach verbindet islamistische und antimuslimische Argumentationen eine Komplizenschaft, die auf der naturalisierenden Kulturalisierung von Differenz beruht und in Rassismus verstrickt ist. Muslimische Gemeinschaften werden in beiden Varianten überislamisiert und »aus konkreten sozialen, historischen, politischen, ideologischen Gebilden mit bestimmten Tendenzen und Perspektiven ${ }^{19} \mathrm{zu}$ einem ahistorischen, essentiellen Islam umgedeutet. Weil Religion als Kultur und beides als unveränderlich und statisch verhandelt wird, spricht Al-Azmeh von Rassismus. Auch Said hatte den kolonialen Orientalismus des 18. und 19. Jahrhunderts als Rassismus theoretisiert. ${ }^{20}$ Aktuelle Fachdebatten stimmen mit dieser Einordnung überein und sprechen von antimuslimischem Rassismus. ${ }^{21}$

Der antimuslimische Rassismus hat Folgen, die über die Wahrnehmung von Muslim:innen als fremd und bedrohlich und die Geringschätzung des Islams und der Muslim:innen hinausgehen. ${ }^{22}$ Im Bedrohungsszenario des sislamistischen Terrorismus` werden (als) Muslim:innen (Markierte) ihrer tatsächlichen oder zugeschriebenen Religion, Kultur und Herkunft nach als spezifische Problemgruppe ins

\footnotetext{
18 Al-Azmeh 1996: 21.

19 Ebd.: 7.

20 Said 1978, 1985.

21 Attia/Keskinkılıç 2016.

22 Hafez/Schmidt 2014.
} 
öffentliche, politische, wissenschaftliche und pädagogische Visier genommen und unter Aufsicht gestellt. Das Bedrohungsszenario mündet in Politiken der Kriminalisierung, Verdächtigung und Überwachung von (als) Muslim:innen (Markierten) als potentielle »Gefährder « ${ }^{23}$. Und das, obwohl »die Geschichte des modernen Terrorismus keine Geschichte islamistischer Gruppierungen« ist und »andere Formen staatlicher wie nicht staatlicher Gewalt ein rechnerisch größeres Gefährdungspotenzial für die innere wie auch internationale Sicherheit $\aleph^{24}$ besitzen. Gleichzeitig wird gegen Muslim:innen gerichtete rassistische und extrem rechte Gewalt individualisiert und marginalisiert ${ }^{25}$ sowie Kritik am antimuslimischen Rassismus verharmlost und diskreditiert. $^{26}$

(Als) Muslim:innen (Markierte) werden mit dem Stigma des bedrohlich Fremden belegt, misstrauisch beobachtet und kommentiert, sie werden der Integrationsverweigerung oder der Integrationsunfähigkeit sowie der Unterwanderung und Islamisierung bezichtigt, sodass ihre Diskriminierung nicht nur als gerechtfertigt, sondern bisweilen gar als geboten erscheint. >Muslim:innen bleiben von hegemonialen Islamdebatten und der damit zusammenhängenden Anrufung als Sicherheitsbedrohung nicht unberührt: Sie sehen sich mit alltäglichen Anfeindungen auf offener Straße konfrontiert, erfahren institutionelle Diskriminierung in Bildungseinrichtungen, im Beruf, auf dem Wohnungsmarkt und im Gesundheitssystem ${ }^{27}$ und müssen sich - ob sie wollen oder nicht - mit jenen Themen auseinandersetzen, die im hegemonialen Islamdiskurs als 'muslimisch und deswegen als problematisch erscheinen. Sie werden dazu angehalten, sich für die Taten anderer $>$ Muslim:innen zu verantworten, von `bösen Muslim:innen $\mathrm{zu}$ distanzieren und sich unaufhörlich als integrierte, aufgeklärte Bürger:innen zu beweisen.

\footnotetext{
23 Bäcker 2020; Denkowski 2014; Graebsch 2019; Jukschat/Leimbach 2019.

24 Bock 2017: 250.

25 Denkowski 2014; Jukschat/Leimbach 2019.

26 Bartel/Liebscher/Remus 2017; Cremer 2017; Naguib 2013.

27 Bayoumi 2009; Elahi/Khan 2017; Lewicki 2016; Mühe 2019.
} 
Ein möglicher Umgang damit ist, die religiösisierende und kulturalisierende Sicht auf Gewalt, die von >Muslim:innen risch $\mathrm{zu}$ kontextualisieren ${ }^{28}$ oder auch muslimische Kritik daran europäischen Leser:innen zugänglich zu machen. ${ }^{29}$ In der vorliegenden Studie werden wir weitere Deutungen und Strategien im Umgang mit dem Bedrohungsszenario und mit der Adressierung von >Muslim:innen Sicherheitsrisiko rekonstruieren.

\section{Die Adressierung von ıMslim:innen als Sicherheitsproblem}

Die folgenden Ausführungen sind das Ergebnis eines Forschungsprojekts, das (als) Muslim:innen (Markierte) fragt, wie sie den Sicherheitsdiskurs wahrnehmen, welche Bedeutung er für sie hat und wie sie damit umgehen. In Einzelinterviews befragt und zu Gruppendiskussionen eingeladen haben wir Akteur:innen, die in Verbänden, Communities oder Gemeinden aktiv sind und die sich selbst als muslimisch verorten oder als solche angesprochen werden. Damit knüpft das Forschungsprojekt an eine Praxis an, die >Muslim:innen nicht nur als besondere Gruppe ver-andert, sondern im Kontext des Sicherheitsdiskurses anspricht.

Belege für die Adressierung von (als) Muslim:innen (Markierten) als Sicherheitsproblem lassen sich in Praxis reflektierender und erforschender Literatur finden. Qasem ${ }^{30}$ schildert, dass er von einer Schule gebeten wird, einen Workshop durchzuführen. Der Auftrag wird damit begründet, dass einige >muslimischeく Schüler:innen »auffälliges Verhalten« zeigen, ein Schüler soll »auf dem Schulhof predige[n]«. »Ich selber beobachtete in meinen Gesprächen während der Workshops jedoch keinerlei besonderen religiösen Eifer bei diesem Schüler, sondern nahm seine mitunter tatsächlich lautstarken Beschwerden über Rassismus vielmehr als aufrichtige Unmutsäußerungen wahr«, so Qasem. 
Dieses Beispiel steht paradigmatisch für viele seiner Erfahrungen im Zusammenhang mit der Islamismusprävention: "Von Rassismus betroffene Jugendliche - und unter ihnen vor allem diejenigen, die diese Erfahrung auch klar als Erfahrung von Rassismus benennen - werden so automatisch als potenziell radikalisierungsgefährdet gelesen«.

Eine Studie von Karakaşoğlu und Wojciechowicz bestätigt diese Schilderung. ${ }^{31}$ Die Autorinnen zitieren einen Schulleiter, der sich gegen eine Referendarin mit Hijab »energisch zur Wehr gesetzt [hat] «, weil er meint, dass »das Kopftuch die demokratisch strukturierte Ordnung der Schule gefährde[t]«. Ein anderer Schulleiter unterstellt eine Praktikantin mit Hijab seiner direkten Aufsicht (im Unterschied zu den anderen Praktikant:innen, die von der Ausbildungskoordinatorin betreut werden) und versetzt sie wöchentlich in eine andere Klasse, wo sie in der letzten Reihe sitzen muss, weil der Schulleiter nicht will, dass die Schüler:innen sie als künftige Lehrerin anerkennen. Die Erfahrung junger Menschen in (außer-)schulischen Bildungskontexten, als Bedrohung und Sicherheitsrisiko adressiert zu werden, reiht sich in ihre allgemeinen Rassismuserfahrungen ein $^{32}$ und betrifft freilich nicht nur Jugendliche. Im Rahmen einer Studie führen Jukschat und Leimbach Interviews im Justizvollzug durch. Sie berichten, wie ihnen "mehrheitlich vermeintlich >radikalisierte Interviewpartner vermittelt wurden, die, wie sich im biografischen Interview und der rekonstruktiven Analyse zeigte, weder demokratiefeindliche Positionen vertraten noch mit islamistischen Akteur:innen sympathisierten «. ${ }^{33}$ In einem Fall stellt sich heraus, dass die als Islamist vorgestellte Person ein Aktivist der PKK ist und im anderen, dass es sich um einen kurdischen Jesiden handelt.

Quantitative Erhebungen und Meinungsumfragen, die (als) Muslim:innen (Markierte) befragen und erforschen, und von Wissenschaft, Politik, Verwaltung, Medien und NGOs in Auftrag gegeben werden, gewinnen in den letzten Jahrzehnten vermehrt an Bedeutung. Sie bilden

31 Karakaşoğlu/Wojciechowicz 2017: 517-518.

32 Mühe 2019; Scharathow 2014, 2017.

33 Jukschat/Leimbach 2019: 15. 
den Korpus einer Untersuchung von Johansen und Spielhaus ${ }^{34}$. Die Autorinnen analysieren Studien, die über zehn Jahre in sieben west- und nordeuropäischen Staaten durchgeführt wurden. Sie fragen danach, welche Vorstellungen darin über Muslim:innen zum Ausdruck kommen: Wer gilt als muslimisch, wie wird die Stichprobe der $>$ Muslim:innen operationalisiert und wie kommt sie zustande? Wer wird als Muslim:in befragt - und wer nicht, welche Themenfelder werden angesprochen, welche Fragen gestellt - und welche nicht, welches Erkenntnisziel liegt den Befragungen zugrunde und wie werden die Ergebnisse präsentiert? Die Befragungen, so ein Ergebnis von Johansen und Spielhaus, greifen auf gesellschaftlich verfügbares Wissen über Muslim:innen zurück und sind ihrerseits an der Produktion von Wissen über Muslim:innen beteiligt, und zwar im Zuge des gesamten Forschungsprozesses, vom Entwurf des Forschungsdesigns und der Zusammenstellung des Samples, über den Erhebungs- und Auswertungsprozess bis hin zur Präsentation der Ergebnisse.

Die wenigen Beispiele zeigen, dass es auch kritische Auseinandersetzungen mit der einseitigen und unterkomplexen Erforschung von >Muslim:innen gibt sowie Studien, die Muslim:innen dazu einladen, eigensinnige Perspektiven einzunehmen, und Abhandlungen, die die fachlichen Kompetenzen von Muslim:innen ernst nehmen. ${ }^{35}$

\section{Eine weitere Studie über ’Muslim:innen`?}

Unsere Studie folgt der Logik, >Muslim:innen`zum Untersuchungsgegenstand $\mathrm{zu}$ nehmen und sie $\mathrm{zu}$ einem problematischen Sachverhalt zu befragen. Da wir die Effekte der Muslimisierung untersuchen wollen, müssen wir >Muslim:innen $<$ befragen. Wir müssen dem Konstruktionsprozess des >muslimischen Sicherheitsproblems` nachspüren, um seine Funktions- und Wirkungsweisen zu verstehen und ihn »kontra-

34 Johansen/Spielhaus 2012, 2018.

35 Spielhaus 2011; Bassiouni 2014. 
punktisch $\ll^{36}$, also sowohl in der hegemonialen Bedeutung und deren Vereindeutigungen und Auslassungen als auch den Widerständen dagegen, lesen zu können. Die Diskursivierung von >Muslim:innen`als Sicherheitsproblem ist ein gesellschaftlicher Rassifizierungsprozess ${ }^{37} \mathrm{mit}$ realen Folgen für (als) Muslim:innen (Markierte). Von einer Konstruktion als einem Prozess der Rassifizierung zu sprechen, bedeutet freilich nicht, dass es keine Menschen gibt, die sich als Muslim:innen verstehen; vielmehr ist damit die naturalisierende Homogenisierung von Menschen gemeint, die sie aufgrund ihrer (vermeintlichen oder tatsächlichen) Herkunft zu kulturell Fremden erklärt, ihre Fremdheit mit der (vermeintlichen oder tatsächlichen) Religionszugehörigkeit begründet und damit die Herabsetzung und Entwürdigung der so zu >Muslim:innen rassifizierten Menschen rechtfertigt.

Der rassifizierende Konstruktionsprozess hat unter anderem zur Folge, dass sich >Muslim:innen $`$ mit Rassismus auf die ein oder andere Weise beschäftigen müssen, auch wenn sie es nicht wollen. Da Rassismus nicht verschwindet, wenn sich Menschen entscheiden, ihn zu ignorieren, muss diese Entscheidung immer wieder neu getroffen werden; so findet die Beschäftigung mit Rassismus also auch dann kein Ende, wenn ihr eine rassifizierte Person aus dem Weg zu gehen versucht: Sie wird weiterhin als $>$ Muslim:in $<$ adressiert und muss sich aktiv dazu oder auch dagegen verhalten. Rassismus zu ignorieren, ist also keine Option, jedenfalls nicht für Menschen, die rassifiziert werden, und nicht in kritischer Absicht. Um Rassismus der Kritik zugänglich zu machen mit dem Ziel, ihn oder zumindest seine Folgen zu minimieren, muss er thematisiert werden.

Eine der Möglichkeiten, Rassismus zu identifizieren, besteht darin, seine Effekte $\mathrm{zu}$ studieren. Insbesondere nicht-intendierter, institutionalisierter, wohlmeinender, paternalistischer Rassismus kann nur schwerlich an den Motiven erkannt werden. Handlungsroutinen etwa können problematisch sein, wenn ihnen eine spezifische Norm (weiß,

36 Said 1994: 51

37 Attia 2009; Shooman 2014. 
christlich, säkular) zugrunde liegt, die partikular ist, aber universalisiert wird; Personen müssen sich nichts weiter dabei denken, wenn sie wie üblich handeln, und dennoch kann der Effekt ausschließen und dem Ausschluss eine rassistische Konstruktion zugrunde liegen. ${ }^{38}$ Grenzüberschreitendes Verhalten, das damit begründet wird, nur das Beste im Sinn $\mathrm{zu}$ haben und helfen $\mathrm{zu}$ wollen, kann in rassistische Diskurse verstrickt sein, ohne dass die so handelnde Person ein rassistisches Motiv benennen könnte. Und doch haben diese Formen von Rassismus Herabsetzung und Verletzung, Barrieren und Ungleichheit, Wut und Rückzug zur Folge.

Antimuslimischer Rassismus (be-)trifft Menschen, die als >Muslim:innen angesprochen und ausgeschlossen, bevormundet und bemitleidet, zurückgewiesen und behindert, beschimpft und beschuldigt werden, der Sicherheitsdiskurs (be-)trifft (als) Muslim:innen (Markierte). Insofern müssen wir >Muslim:innen befragen, wenn wir rassismuskritische Forschung betreiben wollen, auch auf die Gefahr hin, die Konstruktion der Gruppe der >Muslim:innen - jedenfalls vorübergehend - dadurch zu affirmieren, dass wir unsere Gesprächspartner:innen als solche anrufen.

Johansen und Spielhaus kommen in ihrer Analyse zu dem Schluss, dass in der überwiegenden Mehrzahl der Befragungen bereits die Fragestellung und die Auswahl der Gesprächspartner:innen davon ausgehen, dass Muslim:innen fremd und potentiell problematisch sind. Die Themenfelder nehmen folgende Aspekte in den Blick: »integration, belonging and identity, radicalization and security, religiosity and religious practice, political and civic participation, gender and sexuality «. ${ }^{39}$ In Fragestellungen zu Einstellungen, Werten und Praktiken werden die genannten Themenfelder derart miteinander ins Verhältnis gesetzt,

38 Das Neutralitätsgesetz etwa wurde im Zuge der Kopftuch-/Berufsverbote für Kopftuch tragende, muslimische Frauen an staatlichen Schulen erlassen. Die Begründung für das Verbot findet sich im Cesetzestext nicht wieder, seine Folgen für Hijabis sind geblieben. 
dass im Ergebnis die (vermutete) Religionszugehörigkeit von >Muslim:innen als potentielles Integrationshindernis interpretiert wird und die misslungene Integration als potentielles Sicherheitsrisiko. Über den Aspekt der Integration werden also Religion und Sicherheit miteinander verbunden. Da das Thema Integration nur Migrant:innen (und davon nur bestimmte) betriff, werden deutsche Muslim:innen ohne Migrationsbezüge aus Studien über >Muslim:innen ausgeklammert ${ }^{40}$, während Befragte, die sich trotz Adressierung als Muslim:in als atheistisch oder säkular bezeichnen, nicht aus der Stichprobe entfernt werden. ${ }^{41}$ Die Zusammenstellung des Samples und die in den Blick genommenen Fragen stellen die (vermutete) Differenz, die Religion an Kultur und Nation bindet, in den Mittelpunkt - schließlich begründet diese eine eigene Befragung von >Muslim:innen<. Wenig überraschend erscheinen >Muslim:innen dann tatsächlich als fremd bzw. werden als fremd präsentiert - alles andere wäre unspektakulär und würde keine Sonderbefragung rechtfertigen.

Auch wir überlassen den von uns interviewten Personen und Gruppen nicht die Wahl des Themas, sondern haben ein Erkenntnisinteresse. Hierzu bedienen wir uns zwar offener Erhebungsmethoden, laden unsere Gesprächspartner:innen aber zu einem spezifischen, problematisierenden Thema ein. In der vorliegenden Publikation reihen wir keine Zitate aneinander - auch das würde nicht ungefiltert Aussagen der Gesprächspartner:innen wiedergeben, sondern wäre durch uns ausgewählt und angeordnet -, sondern interpretieren die Aussagen kontrolliert und versuchen $\mathrm{zu}$ rekonstruieren: Letztlich treffen wir als Forschungsteam, das ein Forschungsdesign entwirft, Fragestellung und Methoden festlegt, Interviewpartner:innen und Diskussionsteilnehmer:innen einlädt, Passagen auswählt und interpretiert, den gesamten Prozess über Entscheidungen. Wir haben versucht, der Homogenisierung von (als) Muslim:innen (Markierten) entgegenzuwirken, indem wir das Sample der $\mathrm{zu}$ befragenden >Muslim:innen divers zusammenstellen. Der Problematisierung von >Muslim:innen

$40 \quad$ Hernández Aguilar 2018.

41 Spielhaus 2013. 
wiederum begegnen wir, indem wir diese ins Zentrum unserer Studie stellen: Wir bitten unsere Gesprächspartner:innen, über die Bedeutung des Sicherheitsdiskurses für sie zu sprechen. In Einzelinterviews und Gruppendiskussionen geben wir ihnen die Gelegenheit, ihre Wahrnehmungen und Strategien zu explizieren, ihr Muslimischsein angesichts des Bedrohungsszenarios selbst zu definieren und zu verkomplizieren, miteinander auszuhandeln und voneinander abzugrenzen, sodass sie aus der fremdbestimmten Positionierung >Muslim:in heraustreten und in Selbstpositionierungen aus pluralen, intersektionalen, ambivalenten, brüchigen Perspektiven von Muslim:innen sprechen können. Dies ist mit der Hoffnung verbunden, dass sie auch als solche gehört werden.

Die ausgewählten Auswertungsmethoden orientieren sich an einer rekonstruktiven Methodologie. Sie geht davon aus, dass die Wirklichkeit gesellschaftlich konstruiert ist und es Aufgabe der empirischen Forschung ist, "die (sinnhaften) Konstruktionen der Wirklichkeit zu rekonstruieren, welche die Akteure in und mit ihren Handlungen vollziehen $«{ }^{42}$ Wissensbestände, die Handlungen anleiten, sind meist implizit, sodass nicht einfach danach gefragt werden kann. Vielmehr müssen Handlungen, wozu auch Sprachhandlungen gehören, beobachtet und rekonstruiert werden, um die »implizite Klugheit ${ }^{43}$ sichtbar $\mathrm{zu}$ machen. Das Ziel der Rekonstruktion ist es, "soziale Verhältnisse als Sinnzusammenhänge erfassen zu können und auf diese Weise [...] einen verstehenden Nachvollzug sozialen Handelns zu ermöglichen«, unter anderem auch, weil »sich in der Art und Weise, wie die soziale Welt erfahren wird, milieu- oder generationstypische Lebensbedingungen dokumentieren « ${ }^{44}$ können.

(Als) Muslim:innen (Markierte) zu befragen bedeutet also, dass wir davon ausgehen, dass sie ein Set an sozialen Wirklichkeiten und Wissensbeständen teilen, das Rückschlüsse auf ihre Lebensumstände ermöglicht. $\mathrm{Zu}$ rekonstruieren (oder auch auf andere Weise Daten

\footnotetext{
42 Meuser 2018: 207.

43 Bohnsack zit.n. ebd.: 208.

44 Meuser 2018: 207.
} 
auszuwerten) ist daher kein technokratischer Arbeitsschritt, der von jeder beliebigen Person angewendet zum gleichen Ergebnis gelangt. Vielmehr kommt in allen Schritten des Forschungsprozesses zum Ausdruck, welches (Alltags-)Wissen die Forschenden einspeisen und zur Geltung bringen und wie sie selbst in das Thema involviert sind. Die spezifischen sozialen Wirklichkeiten, die spezifischen Wissensbestände und die besonderen Lebensumstände von >Muslim:innen können religiös, kulturell, gesellschaftlich oder historisch perspektiviert werden oder aber genau diese Perspektivierungen hinterfragt, intersektional miteinander und mit weiteren Aspekten verwoben und/oder rassismuskritisch dekonstruiert werden.

Methoden sind Werkzeuge, aber keine bloßen Techniken; sie werden $\mathrm{zu}$ einem spezifischen Erkenntniszweck eingesetzt, theoretisch begründet und (alltags-)theoretisch umgesetzt. Sie werden von gesellschaftlich positionierten Personen entwickelt und genutzt, die auf die ein oder andere Weise in rassistische Diskurse verstrickt sind, insbesondere dann, wenn die Forschenden sich unbeteiligt oder objektiv wähnen und damit ihre spezifische Position, von der aus sie forschen, nicht offen legen.

Unser Forschungsteam setzt sich aus (als) Muslim:innen (Markierten $)^{45}$ zusammen, die unterschiedliche religiöse, sprachliche, geschlechtliche, sexuelle, soziale, migrationsbiographische, disziplinäre und weitere Positionierungen und Perspektivierungen in die Arbeit einbringen. Die zu den Interpretationsgruppen eingeladenen Personen verfügen über diverse Expertisen und intersektionale Rassismuserfahrungen, sodass weitere Perspektiven hinzukommen. Die (als) Muslim:innen (Markierten), die wir für die vorliegende Studie befragen, sprechen wir - im Unterschied zum Großteil der Studien

45 Im Folgenden nutzen wir die Bezeichnungen Muslim:innen, >Muslim:innen und (als) Muslim:innen (Markierte), um (zunächst) zwischen Figur, Anrufung und Identifizierung, zwischen Selbst- und Fremdbezeichnung zu unterscheiden, die sich sowohl auf die Fokussierung auf das eine zentrale Merkmal, das Muslimischsein, bezieht als auch auf die Frage danach, ob diese Zuordnung überhaupt zutrifft. 
über Muslim:innen - nicht als Fremde und schon gar nicht als problematisch, illoyal und bedrohlich, sondern als Teil der Bevölkerung an. Und obwohl wir >muslimische Akteur:innen befragen, zielt unsere Studie nicht darauf, >Muslim:innen als Typ zu konstruieren und auch nicht verschiedene Typen >muslimischer Wirklichkeiten voneinander $\mathrm{zu}$ unterscheiden, sondern stattdessen möglichst vielfältige gouvernementale und insbesondere widerständige Umgangsstrategien mit dem Sicherheitsdiskurs zu rekonstruieren. Unsere Gesprächspartner:innen (sprach-)handeln auf unterschiedliche und häufig widersprüchliche Weisen. Sie suchen - in (selbst-)reflexiven Überlegungen und gemeinsamen Diskussionen - nach Deutungen und Positionen, nach angemessenem Verhalten und zielführenden Handlungen, sie wechseln die Richtung, sowohl im Denken als auch im Handeln. Insofern präsentieren wir als Forschungsergebnis keine Typologien, sondern Deutungen und Strategien, die um Themenspektren kreisen, welche jeweils neu verhandelt werden und zu verschiedenen, sich kreuzenden und auseinander laufenden, bisweilen ambivalenten und dann wieder klaren Positionierungen einladen. 
